Relations industrielles

Industrial Relations

\title{
Robert Gagnon, Louis LeBel et Pierre Verge, Droit du travail,
} 2e éd.

\section{Alain Barré}

Volume 47, numéro 3, 1992

URI : https://id.erudit.org/iderudit/050797ar

DOI : https://doi.org/10.7202/050797ar

Aller au sommaire du numéro

Éditeur(s)

Département des relations industrielles de l'Université Laval

ISSN

0034-379X (imprimé)

1703-8138 (numérique)

Découvrir la revue

Citer ce compte rendu

Barré, A. (1992). Compte rendu de [Robert Gagnon, Louis LeBel et Pierre Verge, Droit du travail, 2e éd.] Relations industrielles / Industrial Relations, 47(3),

573-577. https://doi.org/10.7202/050797ar

Tous droits réservés @ C Département des relations industrielles de l'Universite Laval, 1992
Ce document est protégé par la loi sur le droit d'auteur. L’utilisation des services d'Érudit (y compris la reproduction) est assujettie à sa politique d'utilisation que vous pouvez consulter en ligne.

https://apropos.erudit.org/fr/usagers/politique-dutilisation/ 


\section{RECENSIONS BOOK REVIEWS}

Droit du travail, $2^{e}$ éd., par Robert Gagnon, Louis LeBel et Pierre Verge, Québec, Les Presses de l'Université Laval, 1991, 1065 p., ISBN 2-7637-7179-3

Lorsque nous avons préparé la recension de la première édition de cet ouvrage paru en 1987, nous l'avions alors salué avec beaucoup de satisfaction puisqu'il venait combler le besoin d'une présentation "complète, fouillée et vigoureuse de notre droit du travail». La seconde édition mérite assurément les mêmes éloges. Adoptant, à quelques rubriques près, le plan de la première édition, les auteurs nous livrent une présentation intégrée du droit du travail.

Dans notre recension de la première édition, nous avions relevé que les auteurs prenaient parfois partie avec vigueur sur des questions controversées de notre droit du travail. Nous avions alors noté que les points de vue retenus relevaient d'une profonde compréhension des principes fondamentaux qui soustendent la législation du travail. On nous permettra de souligner deux questions de droit sur lesquelles les thèses développées dans la première édition furent finalement adoptées par la Cour d'appel du Québec... au prix, dans les deux cas, de revirements jurisprudentiels pour le moins spectaculaires.

Ainsi, dans leur première édition, les auteurs avaient clairement rejeté la distinction entre les termes "congédiement" et "licenciement" dans l'interprétation des articles 82 et 124 de la Loi sur les normes du travail, L.R.Q., c. N-1.1. La Cour d'appel du Québec avait semblé avaliser une telle distinction dans l'affaire Donohue c. Simard, [1988] R.J.Q. 2118. Toutefois, dans l'affaire Lamy c. Kraft limitée, C.A. Montréal, n 500-09-001227-875, 12 décembre 1990, D.T.E. 91 T-49, la Cour d'appel, dans ce qui nous apparaît être un virage à $180^{\circ}$, a décidé que la distinction ne tenait plus. S'appuyant sur une longue citation de la première édition du Droit du travail, la Cour d'appel, sous la plume du même juge que dans l'affaire Donohue c. Simard, rejeta nettement la distinction congédiement-licenciement.

A propos de la question des fausses déclarations au stade de l'embauchage, les auteurs, dans la première édition de leur ouvrage, avaient pris le contre-pied de l'arrêt Montréal-Est c. Gagnon, [1978] C.A. 100. Les auteurs reprennent leurs critiques de cet arrêt dans la seconde édition de leur ouvrage (p. 98). II est toutefois intéressant de noter que le 3 février 1992, soit quelques mois après la publication de cette seconde édition, la Cour d'appel a justement renversé sa jurisprudence sur ce point. II s'agit de l'arrêt Maribro Inc. c. Union des employés(ées) de service, local 298-F.T.Q., C.A. Montréal, n 500-09000924-886, 3 février 1992, D.T.E. 92T-248. Désormais, la rupture de la relation individuelle de travail par l'employeur n'est plus qualifiée - comme elle l'avait été en 1978 dans l'affaire Montréal-Est c. Gagnon - de la nullité absolue d'un contrat 
de travail, mais bien de "congédiement" dont l'examen relève de la compétence exclusive du tribunal d'arbitrage non du tribunal de droit commun. En refusant d'examiner cette question comme un problème relatif à l'existence d'un contrat individuel de travail pour vice de consentement et en qualifiant la rupture de "congédiement", la Cour d'appel reconnaît manifestement le bien-fondé des critiques exprimées par les auteurs du Droit du travail.

Dans le cadre de notre recension de la première édition de l'ouvrage, nous avons émis des réserves à propos d'un passage, à la page 120, où les auteurs semblaient favoriser une analyse contractuelle du rapport de travail, notamment dans le droit des rapports collectifs du travail. Les auteurs y affirmaient que "dans le cadre juridique actuel, le lien contractuel continue néanmoins d'être le facteur de rattachement essentiel de tout régime de travail, qu'il soit issu de la négociation, de la loi ou des deux à la fois» et qu'en présence d'une convention collective, "l'explication classique veut que l'entente individuelle subsiste juridiquement, son contenu étant déterminé par la convention collective» (p. 120). Ce passage était, avions-nous souligné, nettement en opposition avec cette autre affirmation selon laquelle le statut de salarié se réalise dès que coexistent dans les faits ses éléments constitutifs (travail subordonné contre rémunération) "sans qu'il soit besoin d'établir l'existence formelle d'un lien contractuel entre l'employeur et le salariém (p. 62). L'affirmation de la page 120 de la première édition était aussi en opposition avec la critique que les auteurs faisaient de l'arrêt Montréal-Est c. Gagnon, ainsi qu'avec le chapitre relatif à la convention collective dans lequel ils rejettent la thèse de l'incorporation des termes de la convention collective dans les contrats individuels de travail et retiennent plutôt la thèse de l'effet réglementaire de la convention collective. Nous constatons avec satisfaction que ce passage de la page 120 de la première édition a été tout simplement éliminé de la seconde édition. L'ouvrage trouve donc plus de cohérence à nos yeux. Les auteurs rejettent donc toute analyse contractuelle du rapport de travail dans l'application de la législation du travail, notamment dans le droit des rapports collectifs du travail.

Il faut noter sur ce point que, depuis la première édition de l'ouvrage, le rejet de l'analyse contractuelle du rapport de travail dans le secteur des rapports collectifs du travail a reçu un appui pour le moins significatif de la Cour suprême dans l'affaire Caimaw C. Paccar of Canada Ltd., [1989] 2 R.C.S. 983. Dans cet arrêt, le juge Laforest a non seulement réaffirmé la règle formulée en 1976 dans la fameuse affaire McGavin Toastmaster à l'effet que le contrat individuel de travail et le droit commun qui le régit n'ont plus leur place lorsque les conditions de travail sont déterminées dans une convention collective, il a aussi étendu la portée de cette conclusion - l'exclusion des règles du droit commun - à l'entretemps des conventions collectives. Selon cet arrêt, l'exclusion des règles du droit commun n'est donc pas limitée par la durée de la convention collective. Certains auraient peut-être avancé l'idée que les contrats individuels de travail réapparaissent à l'expiration de la convention collective. II n'en est rien. L'exclusion des contrats individuels se continue tant que l'obligation légale de négocier subsiste. Or, dans notre droit, cette dernière subsiste jusqu'à la conclusion de la convention collective suivante (p. 503-504), sauf, bien entendu, l'hypothèse de la révocation de l'accréditation. Pour reprendre les paroles du juge Laforest, dans l'affaire Paccar, «le régime juridique établi par le Labour Code [...] ne laisse aucune place à l'application des principes de common law” 
(p. 1008). Bien que les auteurs du Droit du travail ne le disent pas de cette façon, c'est aussi leur compréhension du droit positif. $\grave{A}$ la page 540 , ils n'hésitent pas à affirmer que «sauf dans le cas de prolongation conventionnelle, au moment de l'acquisition du droit de grève et de lock-out, les conditions de travail prévues dans la convention cessent d'avoir effet et l'on tombe dans un "vide juridique" $"$. Faire référence à un "vide juridique", n'est-ce pas là constater qu'au terme des effets de la convention collective, le contrat individuel ne renaît pas ou ne réapparaît pas? II n'y a donc plus de fondement juridique - légal ou conventionnel - pour prétendre à tel ou tel avantage octroyé auparavant lorsque les employés continuent à fournir leur prestation de travail en l'absence de grève ou de lock-out. C'est l'épreuve de force économique à l'état pur. Ce n'est qu'au terme de cette épreuve de force que les parties établiront généralement un régime de travail applicable de manière rétroactive. Encore une fois, soulignonsle, les auteurs ne le disent pas en ces termes, mais une telle compréhension de la nature juridique des rapports de travail s'inscrit parfaitement dans la logique des idées développées dans leur ouvrage. A cet égard, on peut même s'interroger sur la question de savoir pourquoi les auteurs ne se sont pas référés à l'arrêt Paccar lorsqu'ils font état, à la page 542, de ce "vide juridique»: les propos du juge Laforest s'inscrivent parfaitement dans la logique d'un droit des rapports collectifs du travail excluant totalement l'application des règles du droit commun. En d'autres termes, on pourrait dire que la teneur générale de la seconde partie de leur ouvrage sur les rapports collectifs du travail fait nettement voir, pour adapter le dictum du juge Laforest au contexte québécois, que le régime mis en place par le Code du travail du Québec ne laisse aucune place à l'application des règles du droit commun régissant le contrat individuel de travail.

Enfin, nous ne pouvons terminer la présente recension sans faire mention de l'arrêt de la Cour d'appel dans l'affaire Désormeaux c. Centre de réadaptation des jeunes de l'Outaouais, C.A. Montréal, $n^{\circ}$ 500-09-001425-909, 25 avril 1991, D.T.E. 91T-554. Cet arrêt a été rendu quelques mois avant la publication de la seconde édition de l'ouvrage. Toutefois, compte tenu que cette seconde édition reflète l'état du droit positif en date du $1^{\mathrm{er}}$ avril 1991, comme le mentionnent les auteurs dans leur avant-propos, l'arrêt Désormeaux, rendu le 25 avril 1991, n'est logiquement pas mentionné dans l'ouvrage. L'arrêt Désormeaux nous apparaît être en nette opposition avec la critique faite par les auteurs, dans la première édition (p. 617-619) ainsi que dans la seconde édition (p. 705-706) du Droit du travail, de la jurisprudence de la Cour d'appel à propos de l'accès au tribunal d'arbitrage de l'ex-salarié. Selon cette jurisprudence, l'ex-salarié peut saisir la juridiction ordinaire d'une réclamation puisque cette dernière ne constitue pas un grief au sens du Code du travail. La condamnation sans appel, dirions-nous, de cette jurisprudence dans la première édition du Droit du travail (p. 617-619), nous apparaissait tout à fait persuasive. Ainsi, les auteurs y affirmaient que " [...] la définition du grief apportée par le Code du travail ne tient compte que de la nature ou de l'objet de la mésentente [...]. La question de savoir si le bénéficiaire ultime du grief se rattache encore à l'entreprise paraît alors dénuée de pertinence" (p. 618). Dans la seconde édition de leur ouvrage, les auteurs y reprennent textuellement cette critique de la jurisprudence de la Cour d'appel (p. 705-706). Dans l'arrêt Désormeaux, la Cour d'appel, sous la plume du juge LeBel, l'un des auteurs du Droit du travail, ignore cette critique de la jurisprudence relative à l'ex- 
salarié. Un salarié ayant démissionné de son emploi au terme d'un congé en traitement différé d'une année, congé pendant lequel il avait perçu $80 \%$ de son salaire, l'employeur lui réclamait en Cour supérieure le salaire perçu pendant la durée du congé. Toutes les modalités d'octroi du congé et de remboursement du salaire en cas de non retour au travail au terme du congé était précisées à la convention collective applicable. La Cour d'appel confirme la compétence de la Cour supérieure pour entendre l'action de l'employeur. La Cour d'appel, sous la plume du juge LeBel, affirme que «le non-retour de l'employé dans son poste et le défaut de remboursement [...] se situent ici hors du champ d'application temporel et personnel de la convention collective à l'égard de l'ancien salarié» (p. 10-11) et, parlant de ce dernier, la Cour d'appel affirme "son statut de salarié n'existe plus [...]. La compétence arbitrale supposerait le maintien d'un lien temporel ou personnel avec l'unité de négociation et la convention collective» (p. 11). Ces propos sont tout simplement incompatibles avec la position prise par les auteurs du Droit du travail.

Certes, le juge LeBel bénéficie du droit intrinsèque de modifier sa pensée par rapport à un écrit dont il assume la pleine responsabilité au même titre que ses deux coauteurs. Après tout, changer d'idée, n'est-il pas un signe d'intelligence? Toutefois, le juriste, attentif au développement du droit positif, serait sans nul doute en droit de s'attendre à un minimum d'explications. Si le point de vue exprimé aux pages 617-619 de la première édition et aux pages 705706 de la seconde édition du Droit du travail à propos de la compétence arbitrale en rapport avec l'ex-salarié est incorrect en droit, il serait certes possible de le dire franchement avec des arguments de nature juridique à l'appui. Pour l'instant, l'arrêt Désormeaux n'emporte tout simplement pas notre adhésion. Comme l'affirment les auteurs du Droit du travail, la compétence arbitrale dépend exclusivement de la nature ou de l'objet de la mésentente: ale grief ne postule pas le maintien d'un lien d'emploi» (p. 706).

De plus, dans cette seconde édition du Droit du travail, l'exposé du droit positif est complété par un addenda dans lequel les auteurs évaluent l'impact du projet de code civil du Québec sur le droit du travail. Leurs observations ne portent pas sur le Code civil du Québec, tel que sanctionné le 18 décembre 1991 mais bien sur le projet de loi 125 déposé à l'Assemblée nationale en décembre 1990. Toutefois, les observations formulées par les auteurs sont toujours valables en regard du Code civil du Québec finalement adopté. II n'est pas douteux que la communauté juridique, en droit du travail comme dans les autres branches du droit, recherchera - avec avidité, dirions-nous - toute information disponible à propos du Code civil du Québec. Le lecteur appréciera donc grandement les commentaires formulés par les auteurs du Droit du travail à propos des nombreuses questions que provoquera l'entrée en vigueur du Code civil du Québec. La plus fondamentale de ces questions à nos yeux sera de décider si le Code civil du Québec est de nature à faire perdre au droit du travail "son caractère propre" (p. 742). En d'autres termes, nous pourrions formuler la question de la manière suivante: le Code civil du Québec remet-il en cause l'autonomie du droit des rapports collectifs du travail face au droit commun, autonomie affirmée par la Cour suprême dans les arrêts McGavin Toastmaster et Paccar? Les auteurs constatent que «la question est posée, mais la réponse reste incertaine» (p. 742). Pour notre part, notre réponse serait négative, mais 
la présente recension n'est pas peut-être le lieu pour lancer un tel débat. C'est à suivre.

Tout comme dans sa première édition, l'ouvrage comporte divers outils qui facilitent grandement son utilisation: liste des abréviations, bibliographie, index de la législation, index de la jurisprudence, index analytique et table des matières. Par ailleurs, il faut souligner trois améliorations par rapport à la première édition: le papier est plus épais, le caractère d'impression est plus gros et l'impression est plus foncée. Au total, la lecture de la seconde édition est beaucoup plus facile et plus agréable que celle de la première.

Comme l'a été sa première édition, la seconde édition du Droit du travail sera, dans les années à venir, un outil de travail de tout premier ordre autant pour le praticien des relations du travail, juriste ou non, que pour l'étudiant en droit ou en relations industrielles. En fait, si un praticien ou un étudiant devait n'avoir qu'un seul ouvrage de droit du travail dans sa bibliothèque, c'est incontestablement le Droit du travail qu'il devra posséder: indéniablement le point de départ de toute recherche en droit du travail au Québec.

Alain BARRE

Université Laval

Les relations du travail au Québec, par Jean Gérin-Lajoie, Boucherville, Qc, Gaëtan Morin, 1992, 375 p., ISBN 2891054237

Ce livre vise à combler une lacune ressentie depuis longtemps au Québec, en présentant un manuel d'introduction aux relations du travail qui s'adresse aux étudiants débutants en gestion et en relations industrielles. L'auteur veut montrer comment se vivent les relations du travail dans l'entreprise afin d'en faire ressortir l'impact sur la gestion et les enjeux pour les divers acteurs. Pour ce faire, il compare, «tout au long du livre... les relations du travail dans une entreprise syndiquée et une entreprise non syndiquéen. Cet objectif est partiellement atteint et la responsabilité en incombe à la fois à l'auteur et à l'éditeur.

Le manuel se divise en deux parties. La première vise à décrire le système des relations du travail. Elle comporte six chapitres, soit une présentation des données relatives à la syndicalisation, les facteurs menant à la formation d'un syndicat et les origines de l'accréditation, la procédure d'accréditation, les acteurs, l'encadrement juridique de la négociation ou du conflit et les variantes du système (Code canadien du travail, construction, services publics et fonctions publiques).

La seconde partie de l'ouvrage traite de "la pratique" des relations du travail. Celle-ci se définit par les chapitres suivants: le processus de la négociation, la convention collective (grands types de clauses), la procédure de règlement des griefs ("La voix de l'individu") et l'arbitrage des griefs. Un dernier chapitre ("Tour d'horizon et perspectives») s'interroge sur l'avenir du syndicalisme et présente une des avenues possible en matière de nouveaux rapports d'autorité, soit l'expérience Saturn de GM. 